\title{
Protective Effect of Human Mesenchymal Stem Cells on the Survival of Pancreatic Islets
}

\author{
Giulia Fumagalli, ${ }^{1,2}$, Marianna Monfrini ${ }^{1}$, Elisabetta Donzelli ${ }^{1,3}$, \\ Virginia Rodriguez-Menendez ${ }^{1,3}$, Barbara Bonandrini ${ }^{4}$, Marina Figliuzzi ${ }^{4}$, \\ Andrea Remuzzi ${ }^{5}$, Giovanna D’Amico ${ }^{6}$, Guido Cavaletti ${ }^{1,3}$, Arianna Scuteri ${ }^{1,3}$ \\ ${ }^{l}$ Experimental Neurology Unit, School of Medicine and Surgery, University of Milano-Bicocca, Monza (MB), Italy \\ ${ }^{2}$ PhD Program in Neuroscience, University of Milano-Bicocca, Monza (MB), Italy \\ ${ }^{3}$ NeuroMi, Milan Center for Neurosciences, Milano, Italy \\ ${ }^{4}$ Department of Biomedical Engineering, Istituto di Ricerche Farmacologiche Mario Negri IRCCS, Bergamo, Italy \\ ${ }^{5}$ Department of Management, Information and Production Engineering, University of Bergamo, Dalmine (BG), Italy \\ ${ }^{6}$ Centro Ricerca Tettamanti, Clinica Pediatrica, Università Milano-Bicocca, Monza (MB), Italy
}

Background and Objectives: Transplantation of pancreatic islets is an intriguing new therapeutic option to face the worldwide spread problem of Type-I diabetes. Currently, its clinical use is limited by several problems, mainly based on the high number of islets required to restore normoglycaemia and by the low survival of the transplanted tissue. A promising attempt to overcome the limits to such an approach was represented by the use of Mesenchymal Stem Cells (MSC). Despite the encouraging results obtained with murine-derived MSC, little is still known about their protective mechanisms. The aim of the present study was to verify the effectiveness, (besides murine MSC), of clinically relevant human-derived MSC (hMSC) on protecting pancreatic islets, thus also shedding light on the putative differences between MSC of different origin.

Methods and Results: Threefold kinds of co-cultures were therefore in vitro set up (direct, indirect and mixed), to analyze the hMSC effect on pancreatic islet survival and function and to study the putative mechanisms involved. Although in a different way with respect to murine MSC, also human derived cells demonstrated to be effective on protecting pancreatic islet survival. This effect could be due to the release of some trophic factors, such as VEGF and Il-6, and by the reduction of inflammatory cytokine TNF- $\alpha$.

Conclusions: Therefore, hMSC confirmed their great clinical potential to improve the feasibility of pancreatic islet transplantation therapy against diabetes.

Keywords: Type-I diabetes, Pancreatic islets, Mesenchymal stem cells, Soluble factors

Received: July 30, 2019, Revised: October 9, 2019,

Accepted: October 17, 2019, Published online: December 31, 2019 Correspondence to Arianna Scuteri

Experimental Neurology Unit, School of Medicine and Surgery, University of Milan-Bicocca, Via Cadore 48, Monza (MB) 20900, Italy

Tel: +39-0264488296

E-mail: arianna.scuteri@unimib.it

(c) This is an open-access article distributed under the terms of the Creative Commons Attribution Non-Commercial License (http://creativecommons.org/ licenses/by-nc/4.0/), which permits unrestricted non-commercial use, distribution, and reproduction in any medium, provided the original work is properly cited.

Copyright (C) 2020 by the Korean Society for Stem Cell Research

\section{Introduction}

Type 1 diabetes (T1DM) is a worldwide spread chronic metabolic disease deriving from an autoimmune-mediated destruction of $\beta$-cells within pancreatic islets of Langerhans $(1,2)$. The progressive loss of pancreatic $\beta$-cells has as a consequence an insufficient insulin production, and therefore the strong increase of blood glucose level (3), with dramatic systemic effects (4). So far, administration of exogenous insulin through multiple injections repre- 
sents the gold standard therapy, although it is strongly dependent on patient right compliance, and however it is unable to prevent the onset of diabetes long-term side effects, such as nephropathy, neuropathy, and vascular alterations (5). For these reasons, the seek for alternative therapeutic options is still ongoing and, among them, transplantation of human islets represents a very promising alternative to daily insulin injections for patients with T1DM $(3,6)$, being the only treatment theoretically able to offer a physiological glycemic control (1).

Currently however, many factors still limit this therapeutic option, primarily associated with the requirement of multiple donors (generally two or more cadaveric donors), the low islet yield after human pancreas isolation, the limited lifespan of transplanted islets, and the need for chronic immunosuppressive therapies (7).

To bypass such limitations, during the last decade the use of Mesenchymal Stem Cells (MSC) has been proposed due to their peculiar properties. MSC are adult stem cells isolated mainly from bone marrow but also from other tissues such as adipose tissue, skeletal muscle, dermis, human umbilical cord, dental pulp, amniotic fluid and even peripheral blood (8). They exhibit angiogenic, trophic and anti-inflammatory properties, as well as an immunomodulatory activity (9). Moreover, their availability, plasticity and easily expansion in culture on a theoretical base make MSC a very promising treatment option for a wide variety of clinical diseases (10). They could in fact suppress several T-lymphocyte activities limiting inflammation (11) and improve the regeneration process of damaged tissues through their biological properties (12).

Several in vivo studies have confirmed the putative positive role of MSC co-transplantation with pancreatic islets, which resulted able to improve graft functional survival and transplantation in models of streptozotocin induced diabetic rat, or in injured pancreas (13). However, although these positive results, the mechanisms of MSC effects are still under investigation, and by in vitro studies two different hypotheses have been suggested: i) the differentiation into insulin-releasing cells, and/or ii) a paracrine action through the release of soluble factors (14). Since the clinical need is the identification of the possible role of human-derived MSC (hMSC), aim of this study was to verify the feasibility and the soundness of those mechanisms suggested for murine MSC also for clinically relevant hMSC, thus enabling their translation. In particular, we demonstrated that a positive role could be achieved also by hMSC, however with mechanisms different from those observed in murine MSC, focused in particular on trophic, anti-inflammatory factor release and on in- flammation suppression, which can improve islet survival.

\section{Materials and Methods}

\section{Pancreatic islet isolation}

Male Lewis rats (Harlan Laboratories, Italy) 12 weeks of age were used as donors of pancreatic islets. Animal care and treatment were conducted in conformity with the institutional guidelines, in compliance with national (D.L. vo n. 26/2014) and international (European Union Directive 2010/63/UE: Guide for the Care and Use of Laboratory Animals, U.S National Research Council, 1996) laws and policies. The protocol was approved by the Ethic Committee of Mario Negri Institute for Pharmacological Research ( $\left.{ }^{\circ} \mathrm{BG} 01 / \mathrm{C}\right)$. All the experiments were repeated at least three times to validate the results.

Islets were isolated from the pancreas of Lewis rats (body weight 250 300 g), using an automatic procedure. Briefly, the pancreas of anesthetized rats were distended with collagenase P solution (Boehringer-Mannheim, Mannheim, Germany), removed and then loaded into a digestion chamber at $37^{\circ} \mathrm{C}$. When optimum digestion time was reached, the chamber was flushed with $4^{\circ} \mathrm{C}$ Hanks' balanced salt solution (HBSS, Gibson Nitrogen Corporation, Paisley, Scotland) and digested tissue was purified by centrifugation on a Histopaque gradient $(1.077 \mathrm{~g} / \mathrm{ml}$, Sigma, St. Louis, MO). Islets were cultured at $37^{\circ} \mathrm{C}$ in a humidified atmosphere containing 5\% $\mathrm{CO}_{2}$ in RPMI 1640 medium (Life Technologies Italia, Monza, Italy), supplemented with 10\% fetal bovine serum (EuroClone, Pero MI, Italy) (15).

\section{hMSC isolation and characterization}

hMSC used in this work have been provided by San Gerardo Hospital (Monza, Italy). After obtaining informed consent, hMSC were isolated from bone marrow harvested from the iliac crest of healthy donors and characterized as previously described $(16,17)$. In brief, after a bone marrow transplant, collection bags were washed with PBS (Listar Fish, Milan, Italy) to obtain residual bone marrow.

Mononuclear cells were isolated through a density-gradient separation (Ficoll-Hypaque; GE Healthcare, Milan, Italy) and were cultured in Dulbecco's modified Eagle's medium-low glucose (DMEM-LG, Lonza, Verviers, Belgium) supplemented with $2 \mathrm{mM}$ L-glutamine, $100 \mathrm{U} / \mathrm{ml}$ penicillin, $100 \mathrm{mg} / \mathrm{ml}$ streptomycin, $250 \mu \mathrm{g} / \mathrm{ml}$ Fungizone (Lonza), and 10\% defined fetal bovine serum (FBS; Hyclone, Logan, UT, USA). Cells were plated in culture flasks at a high density and were maintained at $37^{\circ} \mathrm{C}$ in a humidified atmosphere containing 5\% $\mathrm{CO}_{2}$. Attached 
cells were cultured until they reached 80\% 90\% confluence and subsequently were detached with trypsin (Lonza) and subcultured at $4 \times 10^{3}$ cells $/ \mathrm{cm}^{2}$. The medium was changed twice a week.

The immunophenotypic characterization of hMSC populations $\left(10^{6}\right.$ cells/tube) was performed using a flow cytometer and FACS Diva software (BD FACScanto ${ }^{\text {TM }}$ FlowCytometer, BD Biosciences, San Jose, CA, USA) (16).

In order to set up the co-cultures, the effect of two different hMSC concentrations was tested on islet survival: since the results obtained were the same with both the doses, all the following experiments were performed using 500,000 cells hMSC.

\section{Co-cultures set up}

Direct co-cultures: hMSC were previously stained with DiI red fluorescent dye $\left(30 \mu \mathrm{g} / \mathrm{ml}, 1\right.$ hour, $37^{\circ} \mathrm{C}$, Molecular Probes Inc., OR, USA), then 500,000 cells were added to approximately 500 pancreatic islets previously stained with Calcein AM green fluorescent dye (4 $\mu \mathrm{M}$, 1 hour, $37^{\circ} \mathrm{C}$, BD Bioscience, Franklin Lakes, NJ, USA) into a low adhesion flask (Corning Inc., NY, USA).

Co-cultures were maintained for 3 weeks in complete RPMI 1640 medium. The medium was changed twice a week. The ability of hMSC to coat pancreatic islets was evaluated by examining the cultures weekly under an inverted microscope.

Indirect co-cultures: hMSC were plated at a density of 500,000 cells/dish onto $35 \mathrm{~mm}$ Petri dishes to which were added approximately 500 pancreatic islets placed in a Transwell insert (BD, San Jose, CA, USA). Co-cultures were maintained for 3 weeks in complete RPMI 1640 medium. The medium was changed twice a week.

Mixed co-cultures: In this type of co-culture, direct and indirect co-cultures co-existed: hMSC were both in direct contact with pancreatic islets in a Transwell (500,000 cells/Transwell) and adherent to the bottom of the dish (500,000 cells/dish). Co-cultures were maintained for 3 weeks in complete RPMI 1640 medium. The medium was changed twice a week.

\section{Assessment of islet viability}

Islet survival was evaluated through a weekly count of floating viable islets, with diameters greater than $150 \mu \mathrm{m}$, under an inverted microscope and islet survival percentage was calculated. Viable islets appeared stained in green with Calcein. Pancreatic islets with diameters lower than $150 \mu \mathrm{m}$ have not been counted.

\section{Assessment of pancreatic islet functionality}

Insulin release detection: Pancreatic islet functionality was assessed weekly by analyzing insulin release modifications after variations of glucose concentration in culture medium. Pancreatic islets cultured alone, co-cultured and hMSC were exposed to a 2 hours pre-incubation with fresh complete RPMI medium containing a low glucose concentration $(1.67 \mathrm{mM})$. At the end of pre-incubation, cultures were exposed to basal medium (1.67 mM glucose) for 1 hour, followed by an incubation with medium supplemented with a high glucose concentration $(20 \mathrm{mM})$, and finally incubated with low glucose concentration (1.67 $\mathrm{mM}$ ) for 1 hour. Medium supplemented with high glucose concentration $(20 \mathrm{mM})$ and medium of the second low glucose concentration incubation $(1.67 \mathrm{mM})$ were collected. Insulin analysis was performed using an EnzymeLinked Immunosorbent Assay (ELISA) kit (Temaricerca S.r.l., Italy) according to the manufacturer's instructions. The assay was able to recognize insulin released by both rat and human species.

The amount of insulin was normalized for the number of islets in each type of culture.

Immunofluorescence analysis: The cultures were pelleted, washed with Phosphate Buffered Saline (PBS) and then fixed in 4\% paraformaldehyde for 1 hour at room temperature. After washing with PBS, cultures were incubated in $20 \%$ sucrose for 12 hours and then embedded in Optimal Cutting Temperature (OCT) medium and frozen in liquid nitrogen. For each sample 10 sections of 10 $\mu \mathrm{m}$ were obtained at a cryostat microtome and analyzed by immunofluorescence studies.

Non-specific binding was blocked with 3\% BSA in PBS for 1 hour, and then samples were incubated overnight at $4{ }^{\circ} \mathrm{C}$ with different primary antibodies according to the manufacturers' protocols: Insulin (Cell Signaling, Danvers Beverly, Massachusetts, $1: 100$ ), the active form of caspase 3 (Cell Signaling, Danvers Beverly, Massachusetts, $1: 500$ ) and 7 (Calbiochem, Darmstadt, Germany, 1 : 100). After washing with PBS, a 1-hour incubation with the secondary antibodies was performed at room temperature (anti rabbit Alexa-Fluor 647, Invitrogen, 1 : 200). Then cells were washed with PBS and coverslips were mounted. Cells were then examined using confocal laser microscopy, carried out with a Radiance 2100 confocal microscope equipped with a krypton/argon laser. Noise reduction was achieved by Kalman filtering during acquisition.

The area of antibody positive cells/islet area was measured with the ImageJ program (NIH, Bethesda, MS, USA), for the quantification of insulin, active caspase 3 and ac- 
tive caspase 7 positive cells, and it was expressed as percentage \pm SD.

\section{ELISA assays for soluble factors}

The supernatants from islets cultured alone and co-cultured with hMSC were collected at the third week of culture and different ELISA assays, able to specifically recognize human samples, were performed in order to detect the trophic factor VEGF (Vascular Endothelial Growth Factor; Sigma-Aldrich, St. Louis, MO), the anti-inflammatory cytokine IL-6 (Interleukine 6; Millipore, Massachusetts, USA) and the pro-inflammatory cytokine TNF- $\alpha$ (Tumor Necrosis Factor alpha; Invitrogen, Frederick, $\mathrm{MD}$ ), according to the manufacturer's instructions.

\section{Statistical analysis}

Values are expressed as mean $\pm \mathrm{SD}$ of three independent experiments. Statistical analysis was performed using the ANOVA test and Tukey's multiple comparison test with the GraphPad Prism (GraphPad Software, San Diego, CA) statistical package. A p value of less than 0.05 was considered statistically significant.

\section{Results}

\section{Effect of different co-culture system}

The first goal of our study was to analyze the effect of different kinds of co-culture between hMSC and pancreatic islets, in order to ascertain the role of a direct interaction as well as the relevance of the soluble trophic factor release. With this aim two experimental models were prepared, a direct co-culture model, with hMSC simply added to floating pancreatic islets, and an indirect co-culture system, represented by dishes with hMSC seeded on the bottom, and a Transwell insert containing floating pancreatic islets. To discriminate between hMSC and pancreatic islet cells during the co-culture, a differential staining was per- formed before the co-culture setup; in particular, hMSC were stained with the vital red fluorescent dye DiI, while pancreatic islets were stained with Calcein AM.

As shown in Fig. 1a, hMSC were able to coat floating pancreatic islets, thus forming three-dimensional floating structures in which pancreatic islet represented the internal core, while hMSC grew in adhesion on the outside. Almost the total part of pancreatic islets resulted completely or partially coated by hMSC. A small percentage of hMSC failed to coat pancreatic islets, being adherent to the bottom of the flask and presenting the particular fibroblastic-like shape, already described (18), and for this reason they were discarded by moving the cellular suspension in a new flask. In this way, a flask with only hMSC coated pancreatic islets was obtained. This co-culture system lasted up to the end of the experiment (3 weeks of culture) and, in this condition, pancreatic islets maintained their roundish morphology until the end of the experiment (Fig. 1b).

Differently, in indirect co-cultures, the presence of the Transwell slightly affected pancreatic islet morphology from the first week of culture, since they lost the classic roundish morphology and were more prone to attach to the Transwell bottom surface (Fig. 1c). Such an effect was observed regardless of the presence of hMSC, since the same islet altered morphology was present also in mixed co-cultures. Also these co-culture methods anyway lasted up to the third week of culture.

\section{Pancreatic islet survival}

As above mentioned, pancreatic islets cultured alone and in co-culture with hMSC were stained with Calcein, a vital fluorescent dye that stains in green living cells, allowing to assess pancreatic islet viability. Survival examinations were conducted by counting the green Calceinstained viable cells at an inverted microscope at different time point (1, 2 and 3 weeks) (Fig. 2a and Fig. 2b).

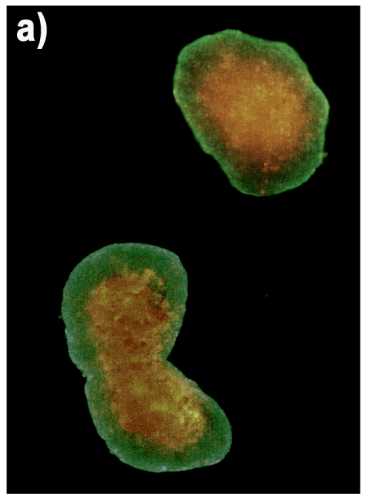

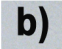

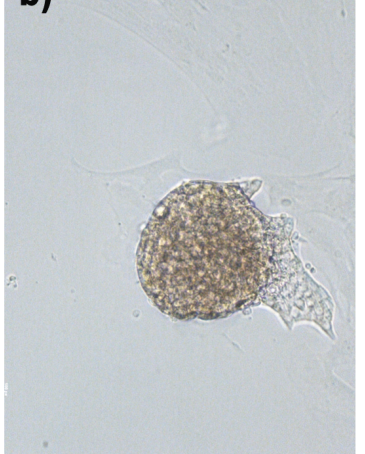

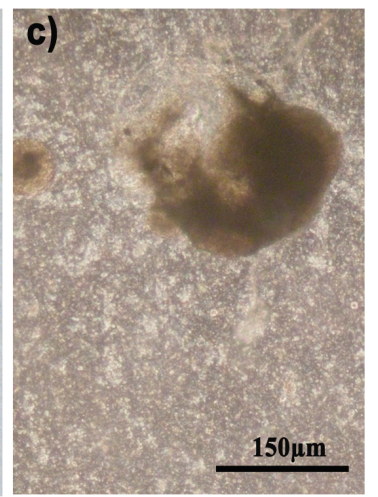

Fig. 1. Islet morphology in co-cultures. (a) 500,000 hMSC were stained in red with the vital fluorescent dye Dil and direct cultured with 500 pancreatic islets stained in green with Calcein dye. hMSC were able to coat pancreatic islets. In green: pancreatic islets. In red: hMSC. (b) Pancreatic islets in directed co-culture with hMSC and (c) pancreatic islets co-cultured indirectly with hMSC at the optical microscope. Bar $150 \mu \mathrm{m}$. 

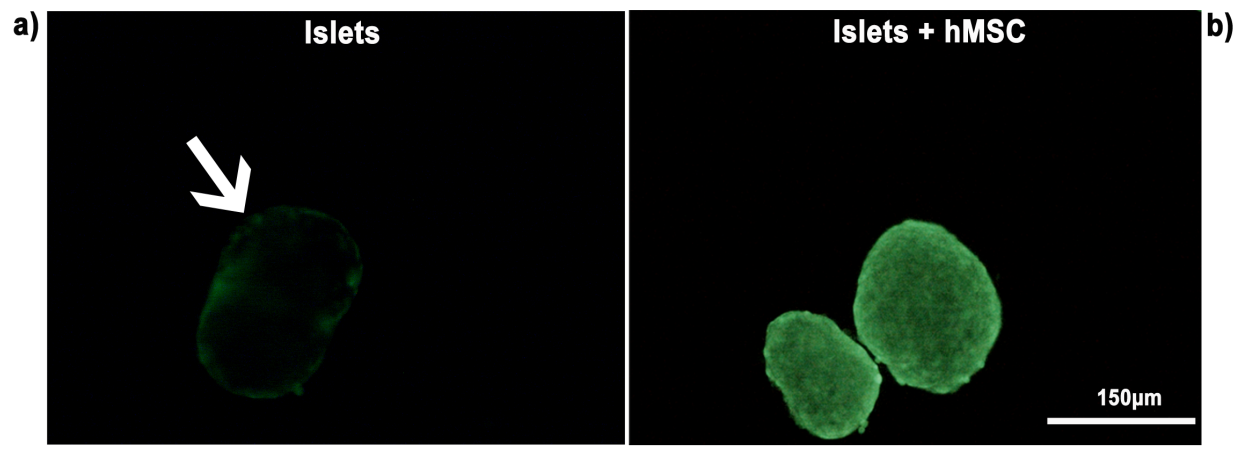

c)

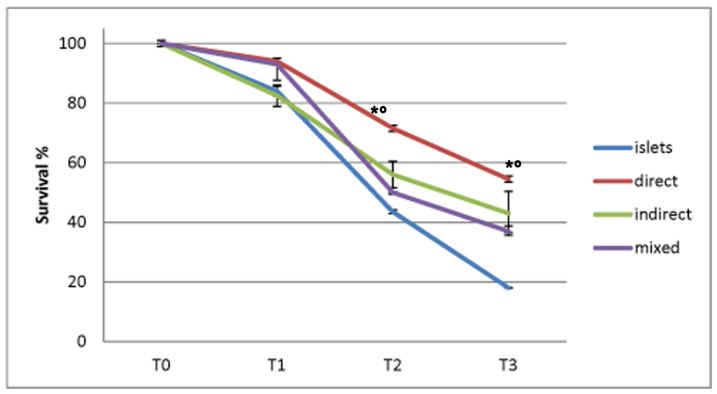

Fig. 2. Survival of pancreatic islets. Pancreatic islet viability was investigated both by observing the presence and distribution of the vital dye Calcein, and by a pancreatic islet count. (a) Representative image of pancreatic islets cultured alone acquired by an inverted microscope: the number of viable cells able to convert the dye in the green fluorescent form is very limited. (b) Representative image of the healthier condition of pancreatic islets cultured in presence of hMSC, irrespective of the co-culture paradigm, showing a uniform spread of the vital dye Calcein up to 3 weeks. Bar $150 \mu \mathrm{m}$. (c) The graph showed the values obtained with the weekly count of viable islets alone or co-cultured. The values are shown as survival percentages and are expressed as the mean \pm SD of three independent experiments. ${ }^{*} p<0.01$ islets vs direct co-cultures, ${ }^{\circ} \mathrm{p}<0.05$ islets vs indirect co-cultures and islets vs mixed co-cultures.

The count evidenced the progressive reduction of survival percentage, up to $20 \%$ after 3 weeks of culture alone, while in co-cultures with hMSC, regardless if direct, indirect or mixed, the count evidenced a statistically significant increase in survival percentage compared to islet alone. In addition, the survival percentage observed after 3 weeks was higher in direct co-cultures (54\%) with respect to indirect and mixed co-cultures, which were comparable (35 40\%) (Fig. 2c).

\section{Cell death evaluation}

As shown in Fig. 2, Calcein spread evenly in islets in direct co-culture with hMSC up to 3 weeks, while in islets cultured alone the arrow indicates that dye distribution was limited to the cells of the outer rim (Fig. 2a).

In order to characterize the cellular death occurring in pancreatic islets during the culture, an immunofluorescence analysis was performed to detect the active form of caspase 3 and 7, pivotal markers of the apoptotic death pathway. As shown in Fig. 3 all the co-culture paradigms resulted negative for the apoptotic markers: the active form of caspase 7 was undetectable in all the samples, while the number of pancreatic islet cells positive to the active form of caspase 3 resulted lower than 5\% when cultured alone, as well as in co-culture with hMSC. The progressive pancreatic islet loss observed during the culture period was therefore ascribable to a necrotic process, while the viability protection offered by all the hMSC co-culture paradigms was not dependent on the well reported anti-apoptotic effect of hMSC, but rather imputable to other molecular mechanisms, likely acting by soluble factors release.

\section{Pancreatic islet functionality}

The pancreatic islet ability to modulate insulin release after glucose variations in the culture medium after the co-culture with hMSC was assessed by a specific ELISA assay, to evaluate if the presence of hMSC could affect the pancreatic islet function.

As shown in Fig. 4, islets both alone and in co-culture with hMSC correctly reacted to the variation of glucose concentration until the third week of culture (Fig. 4): the amount of released insulin increased in presence of a high concentration of glucose and it was reduced after the de- 
a)

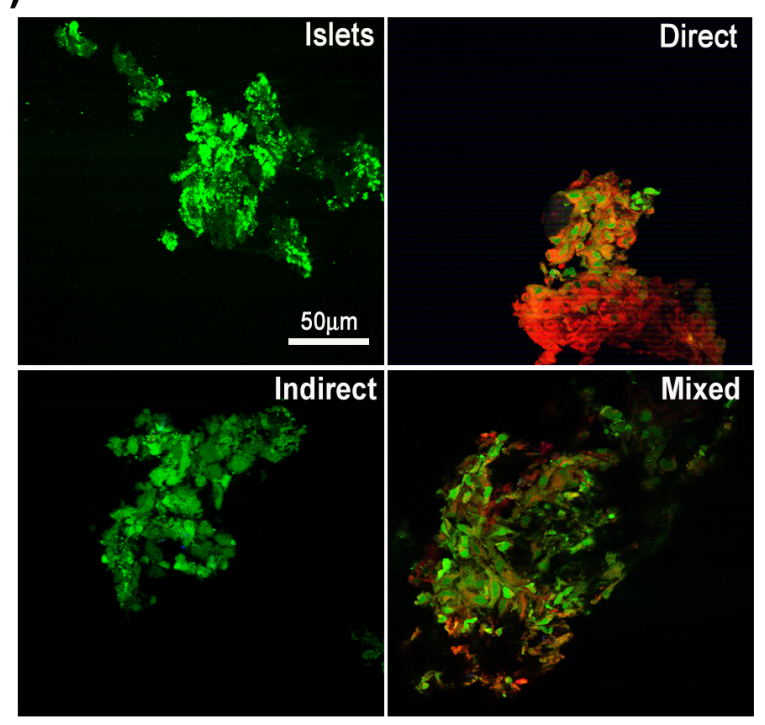

b)

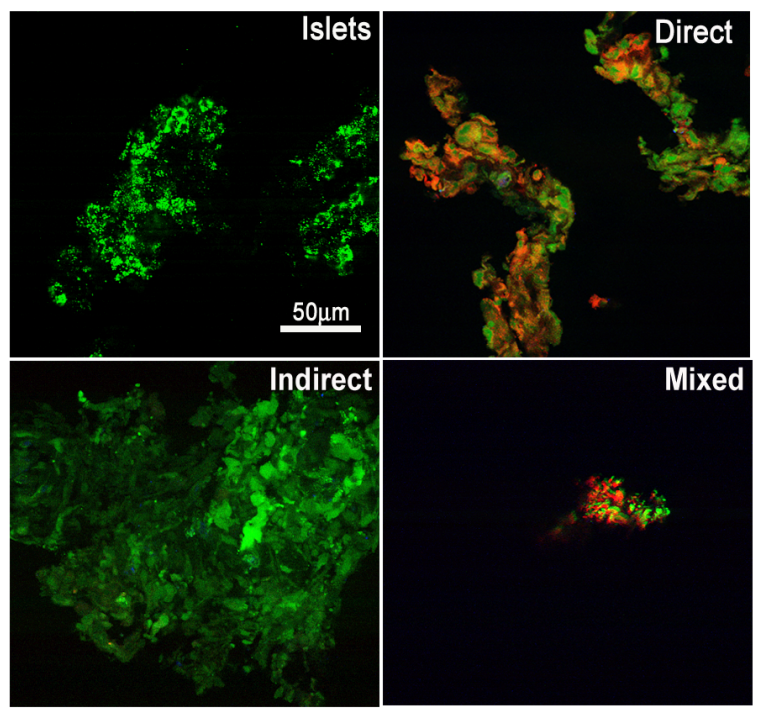

Fig. 3. Apoptosis detection. Pancreatic islets (Islets), direct co-cultures (Direct), indirect co-cultures (Indirect) and mixed co-cultures (Mixed) were analyzed with a specific antibody recognizing the active form of caspase 3 (a) and the active form of caspase 7 (b). In green the pancreatic islets stained with Calcein. In red the hMSC stained with the Dil dye. Bar $50 \mu \mathrm{m}$.

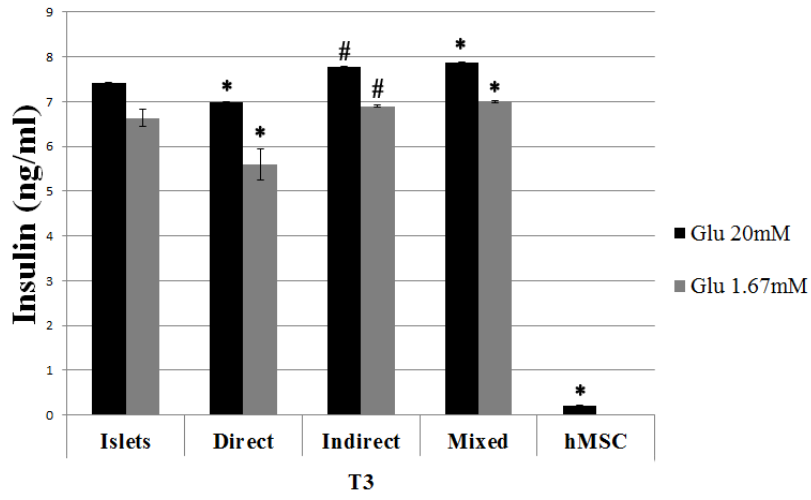

Fig. 4. Insulin release following glucose stimulation after 3 weeks of culture (T3). Islets in culture alone and in co-culture were exposed to different concentrations of glucose in the culture medium (20 mM and $1.67 \mathrm{mM}$ ). After one hour of exposure to each concentration, medium was collected and analyzed by ELISA. The results are expressed as mean \pm SD of three independent experiments. ${ }^{*} \mathrm{p}<$ 0.01 vs islets, $\# p<0.05$ vs islets.

crease of glucose in the culture medium. The presence of hMSC did not affect greatly the islet functionality; actually the insulin release resulted increased in both indirect and mixed co-cultures, thus evidencing a better performance with respect to islets alone, while the insulin level of direct co-cultures was slightly reduced. Anyway, although statistically significant, the differences observed were so little to be biologically irrelevant. In order to as- certain the possible release of insulin by hMSC, an ELISA assay was performed to detect the presence and the amount of insulin into the culture medium of hMSC. As shown in Fig. 4, insulin resulted absent in the culture medium of hMSC cultured alone.

The Immunofluorescence analysis evidenced that insulin was uniformly spread within pancreatic islets alone with a percentage of insulin positive cells of nearly $40 \% \pm 2,4$ (Fig. 5a). Insulin positive staining was also present in pancreatic islets directly co-cultured with hMSC (Fig. 5b) in a similar percentage $(39,83 \pm 3,9)$, as well as indirect $(40,90 \pm 1,9)$ and mixed co-cultures $(45,85 \pm 4,7)$ (Fig. 5d and 5e). It is noteworthy that hMSC didn't result positive to insulin detection in any analyzed sample (Fig. $5 \mathrm{c}$ and $5 \mathrm{f}$ ), thus confirming the ELISA result and suggesting they were therefore not able to directly contribute to insulin production.

\section{Evaluation of the possible mechanism of action of hMSC in co-culture}

Since we observed an increase in pancreatic islet survival after co-culture with hMSC, we investigated the possible role of soluble factors released in medium.

In particular, Vascular Endothelial Growth Factor (VEGF) plays an important role in promoting angiogenesis and structural stability of new vessels (19) and thus maintaining islet functionality (20). MSC are able to promote the release of anti-inflammatory cytokines (e.g. 
Interleukin 6, IL-6) and to inhibit pro-inflammatory ones (e.g. tumor necrosis factor alpha, TNF $\alpha)(21,22)$. Therefore, we focused on the trophic factor VEGF, the anti-inflammatory cytokine IL-6 and the pro-inflammatory cytokine TNF- $\alpha$, and carried out through different ELISA kits a quantitative analysis of their levels in the medium of islets cultured alone, co-cultured and mixed after three weeks of culture.

The analysis evidenced in islets cultured alone the absence of VEGF, which was present on the contrary in pancreatic islets in direct co-culture with hMSC (8248.32 \pm $913.83 \mathrm{pg} / \mathrm{ml})$, as well as in indirect $(7849.25 \pm 108.33 \mathrm{pg} /$ $\mathrm{ml})$ and in mixed co-cultures $(6708.25 \pm 142.4 \mathrm{pg} / \mathrm{ml})$.

Concerning cytokine analysis, TNF- $\alpha$ was present in is-
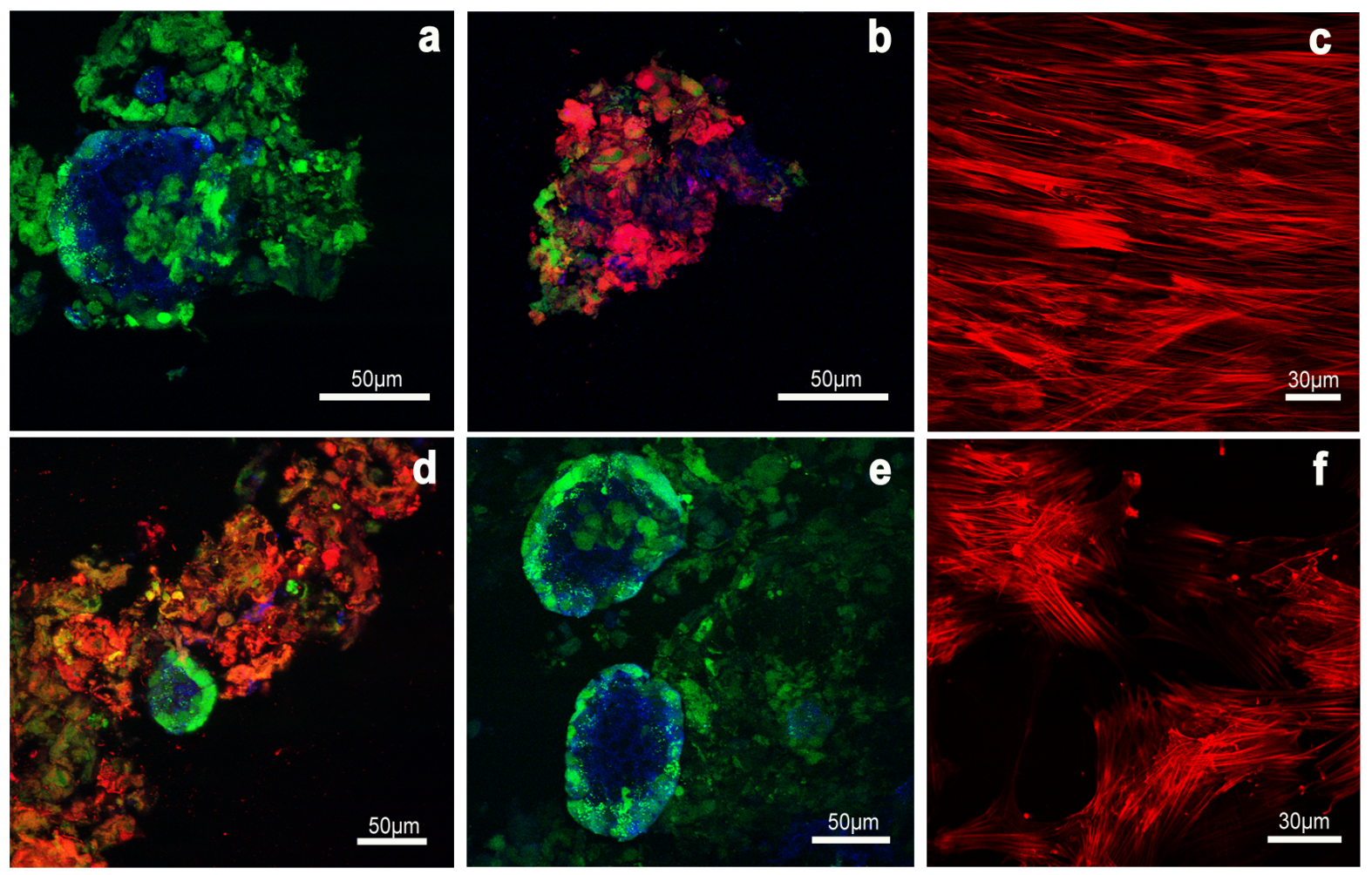

Fig. 5. Evaluation of insulin expression in pancreatic islets alone or in direct co-cultures. Sections from pancreatic islets (a), direct co-cultures (b), hMSC cultured alone (c), mixed co-cultures (d), and indirect co-cultures with islets into the Transwell (e) and hMSC under the Transwell (f) were analyzed at a confocal microscope with a specific antibody for insulin. In green the pancreatic islets stained with Calcein. In red the hMSC stained with the Dil dye. Positive cells for insulin in blue. Bar $50 \mu \mathrm{m}$ and $30 \mu \mathrm{m}$.

a)

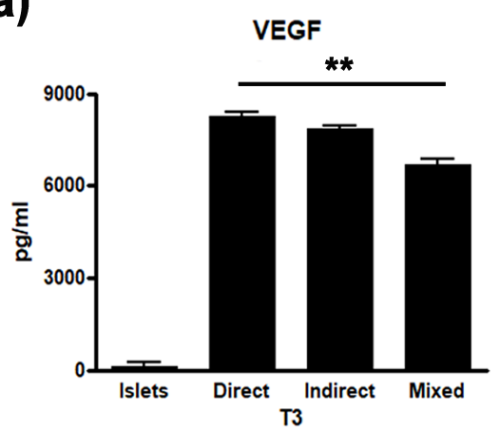

b)

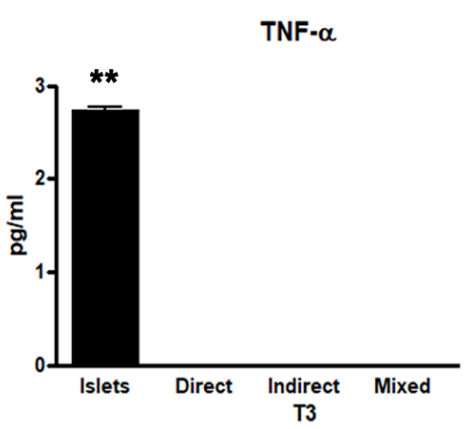

c)

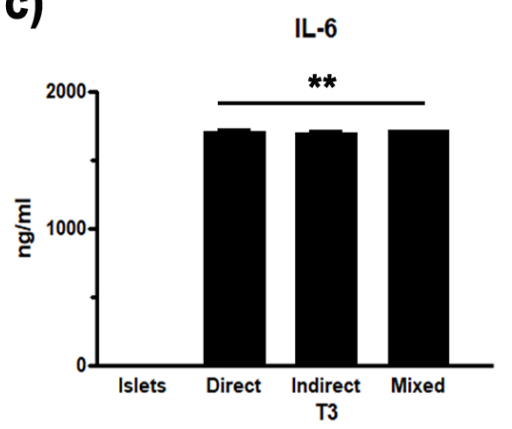

Fig. 6. Release of trophic factor analysis. Release of VEGF (a), TNF- $\alpha$ (b) and IL-6 (c) in medium after 3 weeks of culture (T3). The concentrations were determined by ELISA assay. The results are expressed as mean \pm SD of three independent experiments. ${ }^{* *} \mathrm{p}<0.001 \mathrm{vs}$ islets. 
lets cultured alone $(2.7 \pm 0.52 \mathrm{pg} / \mathrm{ml})$ while it was absent in all the co-culture paradigm (Fig. 6b). On the contrary, IL-6 was absent in islets cultured alone, while it resulted present in the medium obtained from direct co-cultures $(1709.08 \pm 9.1 \mathrm{ng} / \mathrm{ml})$, as well as in in indirect $(1699.5 \pm$ $8.83 \mathrm{ng} / \mathrm{ml})$ and mixed co-cultures $(1712.01 \pm 10.34 \mathrm{ng} / \mathrm{ml})$ (Fig. 6c).

\section{Discussion}

In the present study the effect of human-derived MSC on pancreatic islet survival and function was examined. Pancreatic islet transplantation is a very promising therapeutic alternative to insulin administration for the treatment of type 1 diabetes, currently limited by several important factors $(7,23)$. The co-transplantation with MSC has been suggested in order to improve the clinical applicability of such a method, but despite the promising results (24-26), the exact mechanisms by which MSC are able to improve transplantation efficacy have not yet been understood. In addition, the most part of the papers reported in literature deals with murine $\operatorname{MSC}(14,27,28)$, which use is inapplicable for clinical practice.

In our study, by the setting up of three different types of co-culture conditions (direct, indirect and mixed), we have exploited the ability of hMSC to interact with pancreatic islets, observing their effects both on survival and on function, with the specific aim to evaluate the transfer of the encouraging literature results to clinic, besides shedding light on the effect of MSC derived from different origins $(29,30)$.

As already observed by some authors using rat MSC (18, 31), the direct contact allowed also human-derived MSC to adhere, for the most part, to islet suspension, thus losing their classic fibroblastic-like morphology and organizing into clusters. The presence of hMSC was able to increase the pancreatic islet survival irrespectively they were in direct, indirect or mixed co-cultures, however, the survival percentage resulted higher in direct co-cultures $(50 \%)$, whilst in indirect and mixed co-cultures the survival increase had a lower percentage (35\%). Similar results were collected by Jung and collaborators who demonstrated an increase in survival in direct co-cultures with rat MSC (27). However, these observations were in contrast with the results observed by Scuteri and colleagues in a similar experiment with MSC of murine origin, in which the highest percentage of survival was obtained with indirect co-cultures, while the direct contact was not able to modify the data obtained with islets alone (18).

In our present model, the direct co-cultures seemingly appeared to be the better condition for pancreatic islet survival. In fact, pancreatic islets, in the absence of hMSC, gradually lose their structural integrity, fragmenting over the culture period, while islets directly co-cultured with hMSC maintained their correct morphology, as already reported in literature (27). In the same way, also the distribution of the Calcein vital dye confirmed these observations, especially in direct co-cultures. On the contrary, in indirect and mixed co-cultures, pancreatic islet survival was lower than in direct ones, thus suggesting the need of a direct contact to obtain a better protective effect. Moreover, with regard to mixed co-cultures, in our experiment the two mechanisms (direct contact and factor release) seemed to compete each other, not allowing hMSC to sustain survival, in contrast with what was observed by Scuteri and collaborators, where the survival of the mixed islets was comparable to the indirect ones with the highest percentage (18). An explanation to such an observation could be derived by the fact that, while in the direct co-cultures the islets maintained their classical roundish morphology remaining in suspension, in the indirect and mixed co-cultures the islets tended to assume a more elongated shape and to adhere to the Transwell that separated them from the hMSC. Several studies have underlined the importance of maintaining the correct islet structure in order to ensure their functionality, by preserving their phenotype and secretory properties, the islets have to remain in suspension and not to adhere to a substrate $(28,32)$. In particular, Hou and collaborators have shown that the optimal culture condition maintains the islet three-dimensionality (32). In this way, the lower survival observed in the indirect and mixed co-cultures could be simply interpreted as an artifact: the insert could modify the normal islet morphology, promoting adhesion and thus affecting the survival. However, this effect was not reported by Scuteri and collaborators, who indicated a higher survival in indirect co-cultures (18). These differences are probably related to the different influence of growth factors released by MSC of different origins.

Concerning pancreatic islet function after hMSC co-culture, the differences observed on insulin secretion among the co-culture methods resulted so little to be biologically irrelevant, being also very similar to islets cultured alone. On this point there are counteracting evidences in literature, since some studies observed an increase in insulin release after the direct co-culture, thus suggesting a possible differentiation of MSC in insulin-releasing cells or, at least, an improve of pancreatic islet performance (18, 27, 33). Other studies, however, reported that the insulin release by pancreatic islets was not affected by the direct 
co-culture (34). In our model, the analysis of distribution and release of insulin excluded the differentiation of hMSC into insulin-secreting cells, being negative for insulin expression. The different potential probably could be related to different origins of MSC. Since hMSC were not able to release insulin, our hypothesis was that their effect on islets was not related to an improvement in functionality, but rather to the promotion of pancreatic islet survival, which we observed in all three culture conditions (direct, indirect and mixed co-cultures).

MSC are already known for their anti-apoptotic properties $(35,36)$, but the analysis we performed in our study demonstrated that apoptosis was not involved in pancreatic cell death, as already suggested in literature (37). Anyway, the role of apoptosis or necrosis in pancreatic islets should be further investigated.

Since growth factor release and cytokines could influence pancreatic islet survival $(14,27)$, we focused our attention on the other important ability of hMSC: the release of trophic factors. In particular, on the release of VEGF and on the pro-inflammatory and anti-inflammatory cytokines TNF- $\alpha$ and IL-6, respectively. The important role of VEGF has been already suggested in literature in particular concerning in vivo experiments: VEGF presence could improve islet vascularization and permeability (38). Here we confirmed the positive effect of such trophic factors also for islet survival, since VEGF, absent in islets alone, was present in all the co-culture models, in particular in direct co-cultures, as already reported by other authors using rat-derived MSC (27). Such observation could justify the higher survival rate observed in such experimental model. In the same way, also the anti-inflammatory molecule IL-6 resulted present only in presence of hMSC, as already observed with murine MSC $(14,33)$.

ELISA assay for TNF- $\alpha$ revealed the presence of this pro-inflammatory cytokine exclusively in cultures of islets alone, while its release was greatly reduced in co-culture with hMSC. These results are in agree with those reported in literature in studies using murine MSC (27), thus confirming an action similar to human-derived cells.

More in general, these results confirmed the ability of MSC to regulate the inflammatory response, promoting anti-inflammatory pathways and inhibiting pro-inflammatory pathways, thus increasing the survival. These preliminary results suggested the involvement of soluble factors for the hMSC survival positive effects, however further studies will be necessary to really ascertain the importance of such factors.

Many of the differences observed between our results and other studies reported in the literature could be ascribed to the different origin of the MSC used. In fact, our study denotes the different behavior of MSC of different origins: in similar experimental models, in fact, there were different results depending on the use of MSC of human or murine origin. Several studies have already reported the existence of important differences in genomic stability, in the differentiation and immunoregulatory capacities between MSC of different species (29, 30, 39, 40). These results underline once again the need to validate the results obtained with MSC of murine origin also in models with hMSC, in order to transfer in clinic the results obtained in vitro or in pre-clinical models.

In conclusion, we demonstrated that, differently from rat MSC, hMSC did not differentiate into insulin-releasing cells, underlying a different mechanism of action between the two cell types. Despite that, hMSC, both in direct and in indirect co-cultures, were able to increase pancreatic islet survival, and for this reason they could be used for enhancing the clinic application of pancreatic islet transplantation for diabetes therapy. The increase in survival has been observed in all three types of co-culture set up, and it suggested that such increase can be due to the release of trophic and anti-inflammatory factors by hMSC. The increased survival was particularly remarkable in co-cultures in direct contact with pancreatic islets: although the technical culture conditions with the Transwell could affect the survival of indirect and mixed co-cultures, it should be taken into account that the factors released in the medium could be only partially responsible of hMSC positive effect, and that there are still others molecular mechanisms, to be identified yet. Further studies will therefore have to be conducted to better investigate the mechanism underlying the increase in survival observed when direct contact between hMSC and pancreatic islets is established. In particular, it could be interesting to study the role of miRNA, negative regulators of gene expression, in promoting the hMSC direct contact mediated survival of the pancreatic islets, as well as the effect of hMSC also on pancreatic isles of human source.

\section{Acknowledgments}

We are grateful to Dr. Alessio Malacrida for immunostaining quantification.

\section{Potential Conflict of Interest}

The authors have no conflicting financial interest. 


\section{References}

1. Coronel MM, Stabler CL. Engineering a local microenvironment for pancreatic islet replacement. Curr Opin Biotechnol 2013;24:900-908

2. Johnson PR, Jones KE. Pancreatic islet transplantation. Semin Pediatr Surg 2012;21:272-280

3. Ezquer F, Ezquer M, Contador D, Ricca M, Simon V, Conget $\mathrm{P}$. The antidiabetic effect of mesenchymal stem cells is unrelated to their transdifferentiation potential but to their capability to restore Th1/Th2 balance and to modify the pancreatic microenvironment. Stem Cells 2012;30: 1664-1674

4. Mujica-Mota MA, Patel N, Saliba I. Hearing loss in type 1 diabetes: are we facing another microvascular disease? A meta-analysis. Int J Pediatr Otorhinolaryngol 2018;113:3845

5. Sanlioglu AD, Altunbas HA, Balci MK, Griffith TS, Sanlioglu S. Clinical utility of insulin and insulin analogs. Islets 2013;5:67-78

6. Yeung TY, Seeberger KL, Kin T, Adesida A, Jomha N, Shapiro AM, Korbutt GS. Human mesenchymal stem cells protect human islets from pro-inflammatory cytokines. PLoS One 2012;7:e38189

7. Emamaullee JA, Shapiro AM. Factors influencing the loss of beta-cell mass in islet transplantation. Cell Transplant 2007;16:1-8

8. Chen Y, Shao JZ, Xiang LX, Dong XJ, Zhang GR. Mesenchymal stem cells: a promising candidate in regenerative medicine. Int J Biochem Cell Biol 2008;40:815820

9. Uccelli A, Moretta L, Pistoia V. Mesenchymal stem cells in health and disease. Nat Rev Immunol 2008;8:726-736

10. Murphy MB, Moncivais K, Caplan AI. Mesenchymal stem cells: environmentally responsive therapeutics for regenerative medicine. Exp Mol Med 2013;45:e54

11. Zappia E, Casazza S, Pedemonte E, Benvenuto F, Bonanni I, Gerdoni E, Giunti D, Ceravolo A, Cazzanti F, Frassoni F, Mancardi G, Uccelli A. Mesenchymal stem cells ameliorate experimental autoimmune encephalomyelitis inducing T-cell anergy. Blood 2005;106:1755-1761

12. Battiwalla M, Hematti P. Mesenchymal stem cells in hematopoietic stem cell transplantation. Cytotherapy 2009;11: 503-515

13. Tchokonte-Nana V, Manda JK. Early islets and mesenchyme from an injured adult pancreas improve syngeneic engraftments and islet graft function in diabetic rats. Acta Histochem 2018;120:356-362

14. Park KS, Kim YS, Kim JH, Choi B, Kim SH, Tan AH, Lee MS, Lee MK, Kwon CH, Joh JW, Kim SJ, Kim KW. Trophic molecules derived from human mesenchymal stem cells enhance survival, function, and angiogenesis of isolated islets after transplantation. Transplantation 2010;89: 509-517

15. Remuzzi A, Cornolti R, Bianchi R, Figliuzzi M, PorrettaSerapiglia C, Oggioni N, Carozzi V, Crippa L, Avezza F,
Fiordaliso F, Salio M, Lauria G, Lombardi R, Cavaletti G. Regression of diabetic complications by islet transplantation in the rat. Diabetologia 2009;52:2653-2661

16. Salvadè A, Belotti D, Donzelli E, D’Amico G, Gaipa G, Renoldi G, Carini F, Baldoni M, Pogliani E, Tredici G, Biondi A, Biagi E. GMP-grade preparation of biomimetic scaffolds with osteo-differentiated autologous mesenchymal stromal cells for the treatment of alveolar bone resorption in periodontal disease. Cytotherapy 2007;9:427-438

17. Donzelli E, Lucchini C, Ballarini E, Scuteri A, Carini F, Tredici G, Miloso M. ERK1 and ERK2 are involved in recruitment and maturation of human mesenchymal stem cells induced to adipogenic differentiation. J Mol Cell Biol 2011;3:123-131

18. Scuteri A, Donzelli E, Rodriguez-Menendez V, Ravasi M, Monfrini M, Bonandrini B, Figliuzzi M, Remuzzi A, Tredici G. A double mechanism for the mesenchymal stem cells' positive effect on pancreatic islets. PLoS One 2014;9: e84309

19. Zacharek A, Chen J, Cui X, Li A, Li Y, Roberts C, Feng Y, Gao Q, Chopp M. Angiopoietinl/Tie2 and VEGF/Flk1 induced by MSC treatment amplifies angiogenesis and vascular stabilization after stroke. J Cereb Blood Flow Metab 2007;27:1684-1691

20. Johansson A, Olerud J, Johansson M, Carlsson PO. Angiostatic factors normally restrict islet endothelial cell proliferation and migration: implications for islet transplantation. Transpl Int 2009;22:1182-1188

21. Beyth S, Borovsky Z, Mevorach D, Liebergall M, Gazit Z, Aslan H, Galun E, Rachmilewitz J. Human mesenchymal stem cells alter antigen-presenting cell maturation and induce T-cell unresponsiveness. Blood 2005;105:2214-2219

22. Jiang XX, Zhang Y, Liu B, Zhang SX, Wu Y, Yu XD, Mao $\mathrm{N}$. Human mesenchymal stem cells inhibit differentiation and function of monocyte-derived dendritic cells. Blood 2005; 105:4120-4126

23. Marzorati S, Pileggi A, Ricordi C. Allogeneic islet transplantation. Expert Opin Biol Ther 2007;7:1627-1645

24. Monfrini M, Donzelli E, Rodriguez-Menendez V, Ballarini E, Carozzi VA, Chiorazzi A, Meregalli C, Canta A, Oggioni N, Crippa L, Avezza F, Silvani S, Bonandrini B, Figliuzzi M, Remuzzi A, Porretta-Serapiglia C, Bianchi R, Lauria G, Tredici G, Cavaletti G, Scuteri A. Therapeutic potential of Mesenchymal Stem Cells for the treatment of diabetic peripheral neuropathy. Exp Neurol 2017;288:75-84

25. Figliuzzi M, Cornolti R, Perico N, Rota C, Morigi M, Remuzzi G, Remuzzi A, Benigni A. Bone marrow-derived mesenchymal stem cells improve islet graft function in diabetic rats. Transplant Proc 2009;41:1797-1800

26. Sakata N, Goto M, Yoshimatsu G, Egawa S, Unno $M$. Utility of co-transplanting mesenchymal stem cells in islet transplantation. World J Gastroenterol 2011;17:5150-5105

27. Jung EJ, Kim SC, Wee YM, Kim YH, Choi MY, Jeong SH, Lee J, Lim DG, Han DJ. Bone marrow-derived mesenchymal stromal cells support rat pancreatic islet survival and insulin secretory function in vitro. Cytotherapy 2011; 
13:19-29

28. Karaoz E, Ayhan S, Okçu A, Aksoy A, Bayazıt G, Osman Gürol A, Duruksu G. Bone marrow-derived mesenchymal stem cells co-cultured with pancreatic islets display $\beta$ cell plasticity. J Tissue Eng Regen Med 2011;5:491-500

29. Zavan B, Giorgi C, Bagnara GP, Vindigni V, Abatangelo G, Cortivo R. Osteogenic and chondrogenic differentiation: comparison of human and rat bone marrow mesenchymal stem cells cultured into polymeric scaffolds. Eur J Histochem 2007;51 Suppl 1:1-8

30. Scuteri A, Donzelli E, Foudah D, Caldara C, Redondo J, D’Amico G, Tredici G, Miloso M. Mesengenic differentiation: comparison of human and rat bone marrow mesenchymal stem cells. Int J Stem Cells 2014;7:127-134

31. Wang G, Li Y, Wang Y, Dong Y, Wang FS, Ding Y, Kang $\mathrm{Y}, \mathrm{Xu} \mathrm{X}$. Roles of the co-culture of human umbilical cord Wharton's jelly-derived mesenchymal stem cells with rat pancreatic cells in the treatment of rats with diabetes mellitus. Exp Ther Med 2014;8:1389-1396

32. Hou Y, Song C, Xie WJ, Wei Z, Huang RP, Liu W, Zhang ZL, Shi YB. Excellent effect of three-dimensional culture condition on pancreatic islets. Diabetes Res Clin Pract 2009;86:11-15

33. Karaoz E, Genç ZS, Demircan PÇ, Aksoy A, Duruksu G. Protection of rat pancreatic islet function and viability by coculture with rat bone marrow-derived mesenchymal stem cells. Cell Death Dis 2010;1:e36

34. Duprez IR, Johansson U, Nilsson B, Korsgren O, Magnusson PU. Preparatory studies of composite mesenchymal stem cell islets for application in intraportal islet transplantation. Ups J Med Sci 2011;116:8-17.

35. Wei Z, Chen N, Guo H, Wang X, Xu F, Ren Q, Lu S, Liu
B, Zhang L, Zhao H. Bone marrow mesenchymal stem cells from leukemia patients inhibit growth and apoptosis in serum-deprived K562 cells. J Exp Clin Cancer Res 2009;28: 141

36. Santos Nascimento D, Mosqueira D, Sousa LM, Teixeira M, Filipe M, Resende TP, Araújo AF, Valente M, Almeida J, Martins JP, Santos JM, Bárcia RN, Cruz P, Cruz H, Pinto-do-Ó P. Human umbilical cord tissue-derived mesenchymal stromal cells attenuate remodeling after myocardial infarction by proangiogenic, antiapoptotic, and endogenous cell-activation mechanisms. Stem Cell Res Ther 2014;5:5

37. Ichii H, Wang X, Messinger S, Alvarez A, Fraker C, Khan A, Kuroda Y, Inverardi L, Goss JA, Alejandro R, Ricordi C. Improved human islet isolation using nicotinamide. Am J Transplant 2006;6:2060-2068

38. Hajizadeh-Saffar E, Tahamtani Y, Aghdami N, Azadmanesh K, Habibi-Anbouhi M, Heremans Y, De Leu N, Heimberg H, Ravassard P, Shokrgozar MA, Baharvand H. Inducible VEGF expression by human embryonic stem cell-derived mesenchymal stromal cells reduces the minimal islet mass required to reverse diabetes. Sci Rep 2015;5: 9322

39. Ren G, Su J, Zhang L, Zhao X, Ling W, L'huillie A, Zhang J, Lu Y, Roberts AI, Ji W, Zhang H, Rabson AB, Shi Y. Species variation in the mechanisms of mesenchymal stem cell-mediated immunosuppression. Stem Cells 2009;27: 1954-1962

40. Foudah D, Redondo J, Caldara C, Carini F, Tredici G, Miloso M. Human mesenchymal stem cells express neuronal markers after osteogenic and adipogenic differentiation. Cell Mol Biol Lett 2013;18:163-186 